\title{
A P-graph model for the synthesis of tri-generation systems for multi- period operation
}

\author{
Veronica Gonzales ${ }^{1, *}$, Kathleen Aviso ${ }^{1, *}$, and Raymond Tan ${ }^{1, *}$ \\ ${ }^{1}$ Department of Chemical Engineering, Gokongwei College of Engineering, De La Salle University, 2401 Taft Avenue, \\ Manila 1004, Philippines
}

\begin{abstract}
The progressive development of renewable energy technologies is attributed to the growing energy demand and the depletion of non-renewable resources. Renewable energy technologies are usually characterized by intermittent availability of resources, affecting the capability of energy systems to meet projected energy demands. Tri-generation, or the simultaneous generation of three energy types, becomes a potential solution wherein integration of renewable energy technologies is used to maximize resource efficiency. Synthesizing these systems will require the consideration of techno-economic data and the use of computer-aided techniques to facilitate optimization and design. Mixed-Integer Linear Programming is a commonly used technique for Process Network Synthesis, but its application to complex problems becomes problematic due to numerous alternatives and parameter variations. The Process graph framework offers a less laborious option with its unambiguous representation of process systems, utilizing three algorithms in generating all combinatorially feasible solutions that present the user with both optimal and near-optimal solutions. These become helpful in decision-making especially when factors such as those impossible to be captured mathematically need to be considered. This work presents a P-graph model developed for the synthesis and design of multi-period, biomassfired, tri-generation systems with a case study for power generation in the Philippines to demonstrate its capabilities.
\end{abstract}

\section{Introduction}

Efforts to mitigate global environmental problems have resulted in the development of energy-saving and resource-conserving systems. Polygeneration is one of numerous established and developing technologies that aim to not only conserve already sparingly available resources, but also utilize them to their maximum thermodynamic potential [1]. In doing so, these technologies work to generate two or more energy products, making them appreciably beneficial for areas that are characterized by insufficient resources. However, utilizing these systems to their maximum potential constitutes proper process integration techniques that take into consideration both technical and economic aspects in synthesis and design. Process systems engineering (PSE), or alternatively known as computer aided process engineering (CAPE), employs computer-aided tools in solving process network synthesis and design problems (PNS) that deal with process selection which constitutes complex industrial design [2]. These tools aid in the process of modelling and simulation that effectively determine optimal process networks. $\mathrm{P}$-graph modelling is an approach developed by Friedler et al. $[3,4]$ to serve as a convenient and comparable alternative to conventional Mixed Integer Linear Programming (MILP). It has gained leverage with its unambiguous visual representation of processes and its ability to provide various solution options. The approach employs graph methodology in implementing optimization algorithms, allowing for a less laborious and complex experience in dealing with complex systems [5]. The technique boasts of high computational efficiency in coping with desired process objectives and defining the process system's combinatorially feasible structures [3-5].

Multi-period modelling has been widely explored due to its ability to simulate and depict actual systems that are characterized by real-life scenarios wherein various factors and parameters may vary over given periods. This study demonstrates the capabilities and reliability of a developed P-graph model for a biomass-fired tri-generation system operating under varying conditions and subjected to the effects of seasonal variations.

\section{Problem statement}

The formal problem statement can be stated as follows:

- Given N number of process units to generate M types of product output and to operate during $\mathrm{P}$ periods

- Given that the technical performance of each process unit is known with fixed input and output coefficients

- Given that the availability of resources and the demand for the different product types may vary between operational periods 
The problem is to design the trigeneration system which maximizes the annual profit of the system.

\section{Methodology}

P-graph Studio, the software used for this study, is accessible for free on the website [9]. It was utilized to construct the P-graph model. The new multi-period feature was also utilized to implement the multi-period modelling framework, the process of which will be discussed in the case study presented in the next section.

Prior to the generation of the optimal solution, necessary techno-economic data are required for calibration. The case study presented in this work makes use of actual data in order to represent a true-to-life process. These data were acquired from various literature, manufacturer specifications and newspaper articles and are tabulated in the tables presented below. Table 1 presents the cost functions for the process units considered in this study. The fuel options considered for the tri-generation system are agricultural biomass, hence necessitating suitable prime movers to accommodate their utilization. As such, the prime movers considered to be integrated were the Stirling engine and the Externally Fired Gas Turbine (EFGT), both of which are characterized by an external-firing mechanism that allows for the use of flexible and low-quality fuel [8,10], making them advantageous for rural applications. Additional equipment such as the diesel-generator, biomass boiler, adsorption chiller and absorption chiller were also integrated to serve as supplementary equipment for energy generation. The material costs were also obtained through thorough research and are tabulated in Table 2.

Table 1. Cost functions for integrated process units

\begin{tabular}{cccc}
\hline & \multicolumn{2}{c}{ Cost function values } \\
\cline { 2 - 4 } Process Unit & $\begin{array}{c}\text { Fixed } \\
\text { Cost }(\boldsymbol{\epsilon})\end{array}$ & $\begin{array}{c}\text { Variable Cost } \\
(\boldsymbol{\epsilon} / \mathbf{k W})\end{array}$ \\
\hline Stirling Engine & 1,819 & $9,913.9$ \\
EFGT & 277.44 & 593,773 \\
Biomass Boiler & 231.83 & 150,658 \\
Absorption Chiller & 100.04 & 27,791 \\
Adsorption Chiller & 604.11 & 14,499 \\
Diesel-Generator & 0 & 5.83 \\
\hline \multicolumn{5}{c}{} \\
\hline \multicolumn{5}{c}{ Table 2. Material costs } \\
\hline Material & $\mathbf{\epsilon} / \mathbf{M W h}$ & $\mathbf{\epsilon} / \mathbf{M t}$ & $\mathbf{\epsilon} / \mathbf{m}^{\mathbf{3}}$ \\
\hline Rice Husks & - & 43.98 & - \\
Corn Residue & - & 188.55 & - \\
Heating & 126.55 & - & - \\
Cooling & 162.77 & - & - \\
Electricity & 140.78 & - & - \\
Diesel & - & - & 625 \\
\hline
\end{tabular}

The calibration of the model also necessitates inputoutput flow rates which were determined by use of process unit efficiencies. The data tabulated in Table 3 were obtained from either manufacturer specification sheets or cited in literature case studies.

Table 3. Process unit efficiencies

\begin{tabular}{cccc}
\hline Process Unit & $\boldsymbol{\eta}_{\text {heat }}$ & $\boldsymbol{\eta}_{\text {power }}$ & $\begin{array}{c}\text { Coefficient of } \\
\text { Performance }\end{array}$ \\
\hline Stirling Engine & 0.65 & 0.22 & - \\
EFGT & 0.60 & 0.29 & - \\
Biomass Boiler & 0.70 & - & - \\
Absorption Chiller & - & - & 0.70 \\
Adsorption Chiller & - & - & 0.50 \\
Diesel-generator & 0.33 & 0.34 & - \\
\hline
\end{tabular}

\section{Results and discussion}

The case study chosen for this work is representative of a rural power generation solution in the Philippines and reflects the multi-period aspect as an effect of seasonal variations.

The two fuel options considered for the tri-generation system are two of the major agricultural crops in the Philippines - rice and corn, that are both governed by climate changes which effectively impact their cropping calendars. Consequently, this results in a discontinuous availability of biomass for a hypothetical tri-generation plant. The consideration of two types of agricultural biomass then becomes a logical option for periods wherein the availability of one type of biomass may not be sufficient to meet energy demands. A visualization of this intermittent availability is provided in Fig. 1, wherein the fluctuations represent the statistical amount of biomass residue available for a one-year period, the data for which was acquired from residue calculations of the 2016 corn and rice production forecasts by the Philippine Statistics Authority [11]. The figure also presents the individual availabilities of corn residue and rice husks throughout the year, presenting relatively lean periods and one wherein neither of the two options are available. This has resulted in the consideration of diesel as a supplementary fuel option for the plant in order to ensure that the demands are met even in periods wherein the biomass availability may not be adequate.

An assessment of Figure 1 establishes five periods for a given year where each period is characterized by the availability of feedstock. The first covering the first three months, the second covering April, the third covering the next two months, the fourth covering the month of July and the last spanning the remaining five months of the year. 


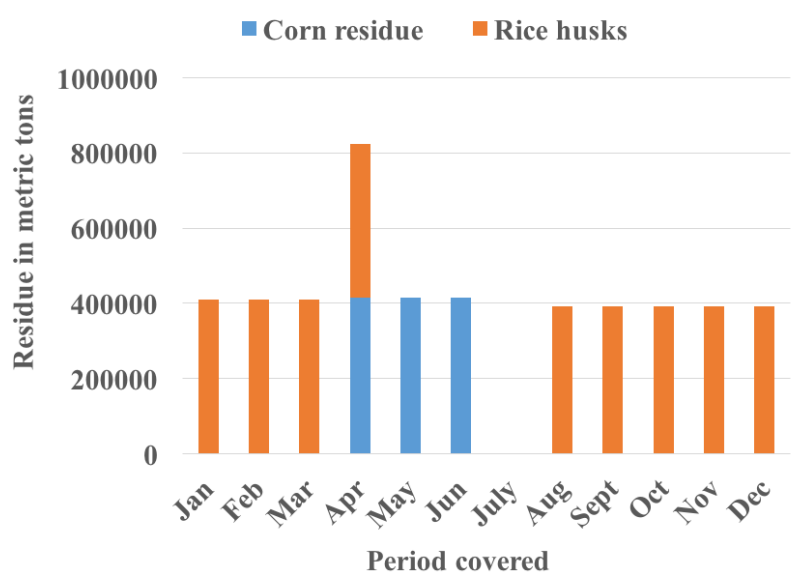

Fig. 1. Residue availability for a one-year period.

This then becomes the five periods considered in this multi-period scenario, and are specified into P-graph's multi-period settings. The periods are represented such that each time period is equivalent to 1 month of an entire year. In this case, a length of 3 is assigned for Period 1, 1 for Period 2, 2 for Period 3, 1 for Period 4 and 5 for Period 5 , which results in a total of 12 months for a period of one year.

Energy demands have also been set to indicate lower and upper bound limits on desired energy products. Although arbitrarily set, the total energy demand for this case study was based off a survey conducted by Foley [6] on rural household energy consumption. Table 4 presents the arbitrary demands for each period.

Table 4. Energy demands for the different periods (in MW)

\begin{tabular}{cccccc}
\hline & \multicolumn{5}{c}{ Period } \\
\cline { 2 - 6 } Prod. & $\mathbf{1}$ & $\mathbf{2}$ & $\mathbf{3}$ & $\mathbf{4}$ & $\mathbf{5}$ \\
\hline Heat & $150-$ & $50-100$ & $100-$ & $50-$ & $250-500$ \\
Space & 2400 & & 200 & 100 & \\
cool. & 360 & $80-120$ & $160-$ & $80-$ & $400-600$ \\
Elec. & $30-60$ & $10-20$ & $20-40$ & $10-20$ & $50-100$ \\
\hline
\end{tabular}

The P-graph model constructed following the encoding of necessary data for calibration is provided in Fig. 2. The rectangular components represent the process units, the concentric circles represent the energy products and the circles with an inscribed triangle represent the fuel options.
The process units filled in black, represent fictitious process units that are to serve as biomass-to-energy converters for the fuel options. This translates to having fuel alternatives, thus providing an option for the system to select a fuel that has more thermodynamic potential.

The data encoded into the multi-period P-graph settings were pre-processed to conform with the multiperiod calculation structure in the P-graph framework as proposed by Heckl et al. [7]. The multi-period structure proposes a representation for physical units operating in multi-period scenarios wherein the required operating capacity of the unit may vary as an effect of irregular energy demands. This representation is illustrated in Fig. 3(b) wherein the demand rates are shown to vary over five periods during the year but collectively add up to a total demand similar to that in Fig. 3(a) for an assumed singleperiod scenario. It is also assumed that in multi-period operations, the rates remain constant throughout a given period. As such, it was discovered that simply doubling the demands for a one-month period to represent an increase in demand for Period 3 (a period covering two months) would be insufficient to define the multi-period scenario. The demands were then modified to suit this analysis such that for an assumed increase in demand for Period 2, an additional factor was multiplied. This factor was to represent the length of the period this increase in demand would cover. Eq. 1 provides an equation for the modification applied, accompanied by a sample calculation.

$$
\text { New demand }=D_{1} \times n
$$

wherein $D_{1}$ represents the demand for a period of length $n$. A sample calculation is done below using the lower bound limit for heat as provided in Table 4. This effectively represents the lower bound limit for heat in Period 3 which has a length of 2, representing the span of two months. Similar calculations were done on the remaining lower and upper bound limits for other energy products to yield the modified boundary limits that were encoded into P-graph.

$$
\begin{aligned}
& \text { New demand }=100 \times 2 \\
& \text { New demand }=200 \mathrm{MW}
\end{aligned}
$$

The cost functions were similarly modified in order to conform with the multi-period calculation structure. Table 5 presents the modified cost functions wherein variable costs were adjusted to represent an average variable cost for a period of one year. 


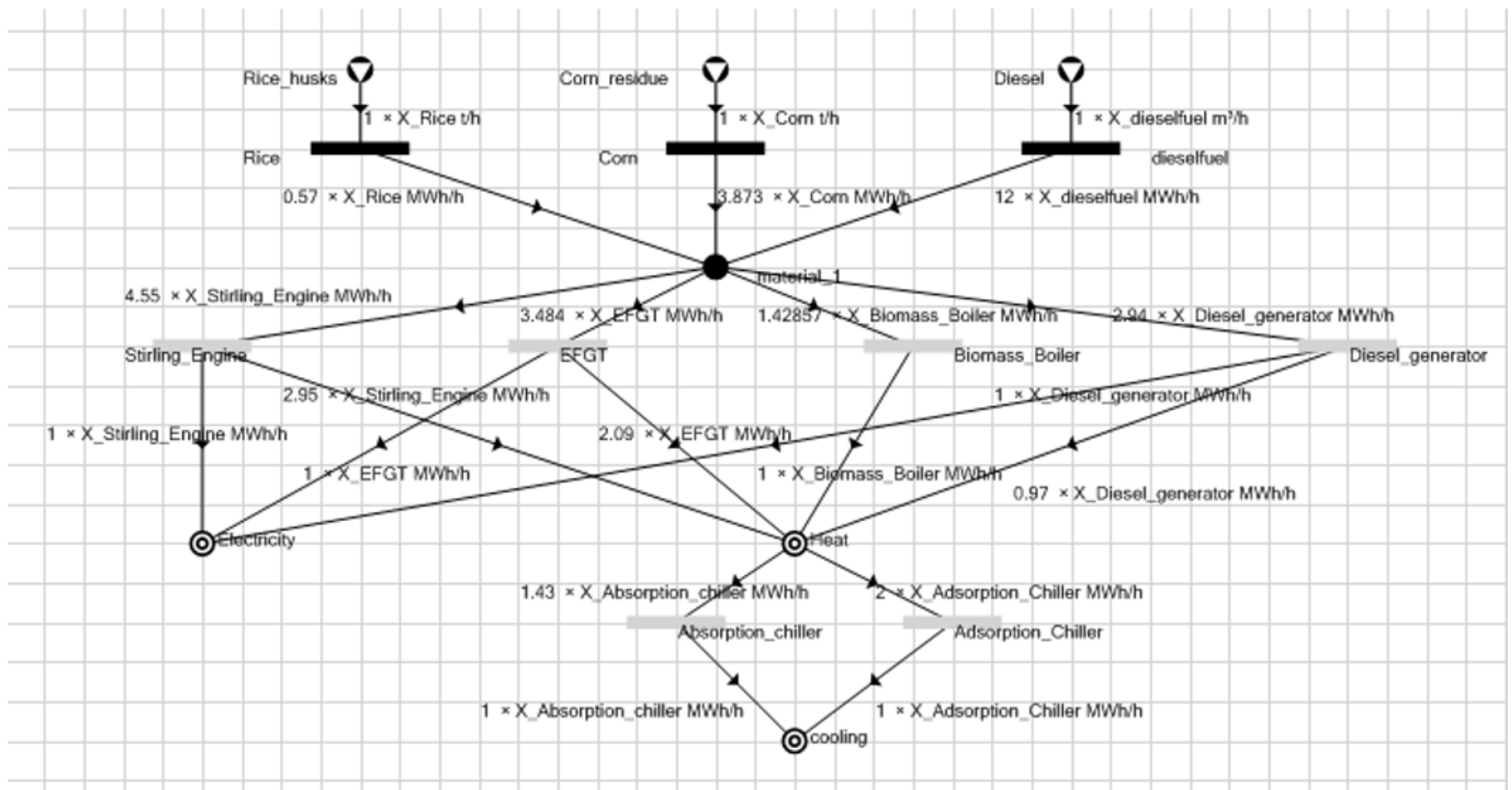

Fig. 2. Constructed P-graph model for the case study

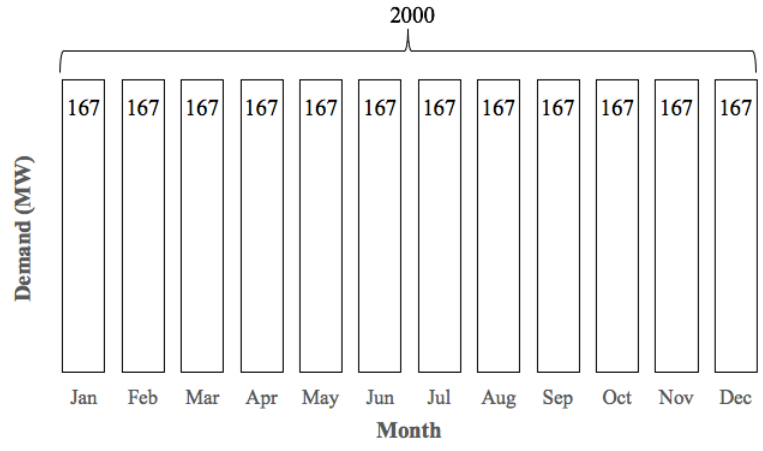

Fig. 3(a). Monthly feed rate for an assumed single operation

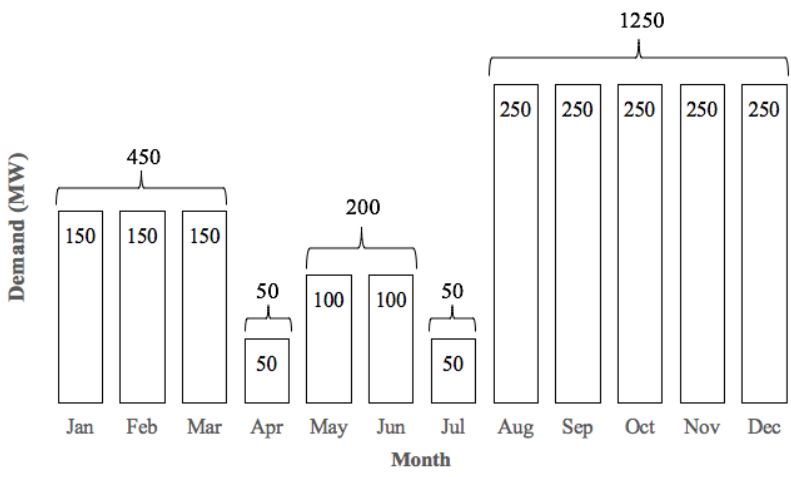

Fig. 3(b). Monthly feed rate for the multi-period operation in the case study

The corrected variable costs were obtained by having divided the original variable costs by 12 whilst the fixed costs were retained so as to reinforce that the acquisition of process units only occurs once whereas the variable expenses are present in the cost calculations throughout the year. The algebraic equation applied in order to derive these values were adapted from the multi-period methodology proposed by Heckl et al. [7]. This is presented as Eq. 2 provided below.

$$
\text { capital cost }=f_{c}+\frac{v c}{p l_{t}}
$$

Wherein $f c, v c$, and $p l_{t}$ represent the fixed cost, variable cost and the total length of the periods considered in the case study.

Table 5. Modified cost functions for integrated process units

\begin{tabular}{cccc}
\hline & \multicolumn{3}{c}{ Cost function values } \\
\cline { 2 - 4 } Process Unit & $\begin{array}{l}\text { Fixed Cost } \\
(\boldsymbol{\epsilon})\end{array}$ & $\begin{array}{c}\text { Variable } \\
\text { Cost } \\
(\mathbf{E} / \mathbf{k W})\end{array}$ & $\begin{array}{c}\text { Corrected } \\
\text { Variable } \\
\mathbf{C o s t} \\
(\mathbf{\epsilon} / \mathbf{k W})\end{array}$ \\
\hline Stirling Engine & 1,819 & $9,913.9$ & 826.16 \\
EFGT & 277.44 & 593,773 & $49,481.08$ \\
Biomass Boiler & 231.83 & 150,658 & $12,554.83$ \\
Absorption Chiller & 100.04 & 27,791 & $2,315.92$ \\
Adsorption Chiller & 604.11 & 14,499 & $1,208.25$ \\
Diesel-Generator & 0 & 5.83 & 0.49 \\
\hline
\end{tabular}

Similar modifications have also been applied to the material costs, a modified table of which is presented in Table 6. Eq. 3 presents the algebraic equation applied to derive the modified material cost values.

$$
\mathrm{p}_{\mathrm{m}}=\frac{\mathrm{p}}{\mathrm{pl}_{\mathrm{t}}}
$$

Wherein $p_{m}, p$, and $p l_{t}$ represent the modified material price, actual price and total length of periods considered in the case study. The optimal process generated by P-graph for the multi-period scenario is tabulated in Table 7. As Pgraph works to allow the consideration of various solution 
options, near-optimal solutions are also generated to provide decision-makers with feasible alternatives. A nearoptimal solution generated for this problem includes the integration of the EFGT for Period 2, effectively affecting the solutions for projected process unit capacities, and the total profit which then amounted to about $47,508.7 € / \mathrm{h}$, nearly $90 €$ less than the estimated profit in the optimal solution presented below. Such variations in process unit selection are influenced by both techno-economic aspects and product demands.

Table 6. Modified material costs

\begin{tabular}{ccccccc}
\hline \multirow{2}{*}{ Material } & \multicolumn{2}{c}{$\boldsymbol{\epsilon} / \mathbf{M W h}$} & \multicolumn{2}{c}{$\boldsymbol{\epsilon} / \mathbf{M t}$} & \multicolumn{2}{c}{$\boldsymbol{\epsilon} / \mathbf{m}^{\mathbf{3}}$} \\
\cline { 2 - 7 } & $\boldsymbol{p}$ & $\boldsymbol{p}_{\boldsymbol{m}}$ & $\boldsymbol{p}$ & $\boldsymbol{p}_{\boldsymbol{m}}$ & $\boldsymbol{p}$ & $\boldsymbol{p}_{\boldsymbol{m}}$ \\
\hline Rice Husks & - & - & 43.98 & 3.67 & - & - \\
Corn & - & - & 188.55 & 15.71 & - & - \\
Residue & & & - & - & - & - \\
Heating & 126.55 & 10.55 & - & - & - & - \\
Cooling & 162.77 & 13.56 & - & - & - & - \\
Electricity & 140.78 & 11.73 & - & - & - \\
Diesel & - & - & - & - & 625 & 52.08 \\
\hline
\end{tabular}

A verification of the results generated by P-graph was carried out using LINGO, a software that implements MILP models equivalent to P-graph.

Table 7. Optimal solution results

\begin{tabular}{ccc}
\hline & Profit generated: 47,596.10 $\mathbf{~ / h}$ & \\
\hline Period & Process Unit Activated & Capacity Multiplier \\
\hline Period 1: $\mathbf{n}=\mathbf{3}$ & Absorption Chiller & 1080 \\
& Boiler & 1913.4 \\
& Stirling Engine & 180 \\
Period 2: $\mathbf{n = 1}$ & Diesel & 296.04 \\
& Absorption Chiller & 120 \\
& Biomass Boiler & 212.6 \\
Period 3: $\mathbf{n = 2}$ & Corn & 101.91 \\
& Stirling Engine & 20 \\
& Absorption Chiller & 480 \\
Period 4: $\mathbf{n = 1}$ & Biomass Boiler & 850.4 \\
& Corn & 407.66 \\
& Stirling Engine & 80 \\
& Absorption Chiller & 120 \\
& Boiler & 212.5 \\
Period 5: $\mathbf{n}=\mathbf{5}$ & Diesel & 32.89 \\
& Stirling Engine & 20 \\
& Absorption Chiller & 3000 \\
& Boiler & 5315 \\
& Diesel & 822.32 \\
& Stirling Engine & 500 \\
\hline
\end{tabular}

The results were similar, thus validating the reliability of the constructed P-graph model. A summary of the Pgraph optimization solution is presented in Table 8.

Table 8. Summary of P-graph optimization results

\begin{tabular}{lc}
\hline & P-Graph Results (€/hour) \\
\hline Total Cost & $48,707.64$ \\
Annualized Capital Costs & $1,111.36$ \\
Total profit & $47,596.28$ \\
\hline
\end{tabular}

\section{Conclusion}

A P-graph methodology for the synthesis of biomass-fired tri-generation systems has been developed in this work. The approach explored the application of graph theory as an alternative method to conventional MILP optimization, the former of which has proven to be advantageous in the synthesis of complex systems. Calibration of the model was carried out with the use of actual data. The computational efficiency and unambiguous representation of process systems contribute to the overall advantage of P-graph methodology. In addition to these, the methodology presents an added user benefit that allows the consideration of multiple solution options, providing the decision-maker with the opportunity to weigh out factors objectively. Several studies exploring its application has also proven the methodology reliable in representing processes affected by multi-period scenarios that characterize actual systems. With the successful validation of the model, the methodology is rendered valid, and can thus be applied to various multi-period systems with quantifiable variations.

Future work could be applied in identifying how other external factors such as climate or economic trends could constitute a multi-period process, provided the effects of said factors are quantifiable. Various modelling techniques can also be tested against P-graph in order to explore its similarities and differences with different software.

This study is a part of the CHED PHERNet project on Low Carbon Energy Systems.

\section{References}

[1] Serra, L. M., Lozano, M.-A., Ramos, J., Ensinas, A. V., \& Nebra, S. a. (2009). Polygeneration and efficient use of natural resources. Energy, 34(5), 575-586. http://doi.org/10.1016/j.energy.2008.08.013

[2] Stephanopoulos, G., \& Westerberg, A. W. (1981). A Review of Process Synthesis. American Institute of Chemical Engineers, 27(3), 321-351

[3] Friedler, F., Tarján, K., Huang, Y. W., \& Fan, L. T. (1992a). Graph-theoretic approach to process synthesis: axioms and theorems. Chemical Engineering Science, 47(8),19731988.http://doi.org/10.1016/00092509(92)80315-4

[4] Friedler, F., Tarján, K., Huang, Y. W., \& Fan, L. T. (1992b). Graph-theoretic approach to process synthesis: axioms and theorems. Chemical Engineering Science, 47(8), 1973$1988 . \quad$ http://doi.org/10.1016/0009. 2509(92)80315-4

[5] Friedler, F., Tarjan, K., Huang, Y. W., \& Fan, L. T. (1992). Combinatorial algorithms for process synthesis. Computers \& Chemical Engineering, $\quad 16, \quad$ S313-S320. http://doi.org/10.1016/S0098-1354(09)800379

[6] Foley, G. (1994). Photovoltaic Applications in Rural Areas of the Developing World (pp. 3536).

[7] Heckl, I., Halasz, L., Szlama, A., Cabezas, H., \& Friedler, F. (2015). Process synthesis involving multi-period operations by the $\mathrm{P}$ graph framework. Computers and Chemical Engineering, 83, 157-164. http://doi.org/10.1016/j.compchemeng.2015.0 4.037 
[8] Kautz, M., \& Hansen, U. (2007). APPLIED for decentralized use of biomass, 84, 795-805. http://doi.org/10.1016/j.apenergy.2007.01.010

[9] Available online at pgraph.dcs.uni-pannon.hu/

[10] Wu, D. W., \& Wang, R. Z. (2006). Combined cooling, heating and power: A review. Progress in Energy and Combustion Science, 32(5-6), 459-495.

http://doi.org/10.1016/j.pecs.2006.02.001

[11] Major Crops Statistics of the Philippines. (2015) 